\title{
Role of Bitter Taste Receptors in Regulating Gastric Accommodation in Guinea Pigs
}

\author{
(1) Yumi Harada, Junichi Koseki, Hitomi Sekine, (DNaoki Fujitsuka, and (DHiroyuki Kobayashi \\ Tsumura Kampo Research Laboratories, Tsumura \& Co., Ibaraki, Japan (Y.H., J.K., H.S., N.F.) and Center for Advanced Kampo \\ Medicine and Clinical Research, Juntendo Graduate School of Medicine, Tokyo, Japan (H.K.)
}

Received January 10, 2019; accepted April 4, 2019

\section{ABSTRACT}

Taste stimulants play important roles in triggering digestion and absorption of nutrients and in toxin detection, under the control of the gut-brain axis. Bitter compounds regulate gut hormone secretion and gastrointestinal motility through bitter taste receptors (TAS2Rs) located in the taste buds on the tongue and in the enteroendocrine cells. Gastric accommodation (GA) is an important physiologic function. However, the role of TAS2R agonists in regulating GA remains unclear. To clarify whether GA is influenced by bitter stimulants, we examined the effect of TAS2R agonist denatonium benzoate (DB), administered intraorally and intragastrically, by measuring the consequent intrabag pressure in the proximal stomach of guinea pigs. Effects of the Kampo medicine rikkunshito (RKT) and its bitter components liquiritigenin and naringenin on GA were also examined. Intraoral DB $(0.2 \mathrm{nmol} / \mathrm{ml})$ administration enhanced GA. Intragastric DB administration (0.1 and $1 \mathrm{nmol} / \mathrm{kg}$ ) promoted GA, whereas higher DB doses $(30 \mu \mathrm{mol} / \mathrm{kg})$ inhibited it. Similar changes in GA were observed with intragastric $(1000 \mathrm{mg} / \mathrm{kg})$ and intraoral $(200 \mathrm{mg} / \mathrm{ml})$ RKT administration. Liquiritigenin and naringenin also promoted GA. These findings suggest that GA is affected by the stimulation of TAS2Rs in the oral cavity or gut in guinea pigs.

\section{Introduction}

Human beings can distinguish between five basic taste qualities-sweet, sour, bitter, salty, and umami (Barretto et al., 2015). Each taste is detected by taste receptor cells assembled into taste buds on the tongue. Hence, taste receptors play a prominent role in taste discrimination. Taste stimulants increase gastric acid and gastrin secretion through the oropharyngeal taste receptors by cephalic phase responses and induce the activation of digestive functions (Katschinski, 2000). In addition, taste receptors are also expressed in the gut and regulate appetite and gastrointestinal (GI) functions (Depoortere, 2014). Sensing the luminal content through taste receptors is important to initiation of appropriate responses to digestion and absorption of nutrients, which are regulated via the gut-brain axis (Daly et al., 2012; Janssen and Depoortere, 2013). Among the taste receptors, the taste 2 receptor family (TAS2Rs) is involved in the perception of bitter compounds (Chandrashekar et al., 2000). Twenty-five TAS2R subtypes have been identified in humans and are considered as playing a role in the detection of toxins. These receptors also affect the secretion of gut hormone and regulate GI motility (Wu et al., 2002; Shi et al., 2003; Chen et al., 2006; Rozengurt and Sternini, 2007; Hao et al., 2008; Jeon et al., 2008; Janssen et al., 2011; Daly et al., 2013).

H.K. received a research grant from Tsumura \& Co. Y.H., J.K., H.S., and N.F. are employed by Tsumura \& Co. All authors declare that they have no conflicts of interest.

https://doi.org/10.1124/jpet.118.256008.
Gastric accommodation (GA) is an essential physiologic function, which allows the stomach to accommodate large amounts of food by reflex relaxation of the proximal stomach during food intake (Tack et al., 1998; Tack et al., 2002; Kindt and Tack, 2006). However, the association between GA and TAS2Rs expressed in the taste buds or GI tract remains poorly understood. In this study, we determined the effects of intraoral (i.o.) and intragastric (i.g.) administration of the bitter agonist denatonium benzoate (DB) on GA in guinea pigs.

Impaired GAs have been reported to be associated with the relaxation of the fundus and are considered a therapeutic target in functional dyspepsia (FD) (van den Elzen and Boeckxstaens, 2006). The Japanese traditional medicine rikkunshito (RKT) is used to treat gastric symptoms in FD patients (Tominaga et al., 2018) with GA impairment (Kusunoki et al., 2010; Shiratori et al., 2011; Miwa et al., 2016). It has been reported that some flavonoids in RKT have agonistic activity toward TAS2Rs (Roland et al., 2013). Therefore, the effects of RKT and these flavonoids on GA in guinea pigs were also examined.

\section{Materials and Methods}

Animals. Four-week-old male Hartley guinea pigs were purchased from Japan SLC, Inc. (Hamamatsu, Japan). During the experimental period following a week of acclimation, they were housed in a regulated environment, with controlled conditions of room temperature $\left(23 \pm 3^{\circ} \mathrm{C}\right)$, humidity $(50 \% \pm 20 \%)$, and lighting (12-hour lightdark cycle). Animals were provided with standard laboratory chow

ABBREVIATIONS: DB, denatonium benzoate; DW, distilled water; FD, functional dyspepsia; GA, gastric accommodation; GI, gastrointestinal; i.g., intragastric (ally); i.o., intraoral (ly); RKT, Rikkunshito; TAS2R, bitter taste receptor. 
and water ad libitum. All experiments were approved by and conducted according to the guidelines of the Experimental Animal Ethics Committee of Tsumura \& Co. (Ibaraki, Japan, approved protocol nos. 10-020, 12-008, and 13-042).

Test Samples. The following test samples were used: TAS2Rs agonist DB (Sigma-Aldrich Co. LLC, St. Louis, MO), Japanese traditional medicine RKT (Tsumura and Co., Tokyo, Japan), liquiritigenin (Wako Pure Chemical Industries, Ltd., Osaka, Japan), and naringenin (Sigma-Aldrich). RKT is a powder extract prepared by mixing extractions from Glycyrrhizae radix, Zingiberis rhizoma, Atractylodis lancea rhizoma, Zizyphi fructus, Citri unshiu pericarpium, Ginseng radix, Pinelliae tuber, and Poria in hot water, followed by spray drying. The quality, efficacy, and safety of the drugs were evaluated by the Pharmaceuticals and Medical Devices Agency (PMDA) (available at https://www.pmda.go.jp).

Animal Preparation. Guinea pigs are widely used for studying gastric contraction and motility (Ji et al., 2003; Cellini et al., 2011; Tsai et al., 2018). The methods to evaluate GA in the conscious guinea pig have been previously established (Koseki et al., 2012; Youn et al., 2015; Miwa et al., 2016; Ikeo et al., 2017). Briefly, a balloon-like apparatus, composed of a polyethylene bag (maximum volume $14 \mathrm{ml}$, thickness $0.01 \mathrm{~mm}$ ) connected to a polyethylene tube (PE 60; Becton, Dickinson and Company, Franklin Lakes, NJ) was prepared before the surgery. Guinea pigs food-deprived for over 16 hours were anesthetized with sodium pentobarbital $(30 \mathrm{mg} / \mathrm{kg}$, i.p.; Kyoritsu Seiyaku Corporation, Tokyo, Japan) and were maintained under anesthesia by isoflurane inhalation for about 30 minutes. After abdominal laparotomy to expose the stomach, a small incision was made near the distal part of the stomach, and residual stomach content was removed. The polyethylene bag of the balloon-like apparatus was deflated and inserted into the proximal stomach from the distal part and was left in the fundus part. The polyethylene tube was then withdrawn from the inside of the stomach through the incision at the distal part and sutured and fixed together with the incision. The polyethylene tube was then subcutaneously threaded through the abdominal wall of the right flank and emerged from the back of the neck. All incisions were closed surgically with sutures. This is illustrated in the report by Youn et al. (2015). The measurements were performed under conscious conditions for 5-28 days after polyethylene bag placement.

Measurement of Liquid Meal-Induced GA. GA was measured in conscious animals as described in a previous report (Koseki et al., 2012). During measurement, conscious guinea pigs were placed in a small transparent plastic box. The polyethylene tube emerging from the back of the neck was connected to a pressure transducer (MLT0699; AD Instruments Pty. Ltd., Bella Vista, NSW, Australia) and a syringe pump (KDS-200; KD Scientific Inc., Holliston, MA) to inject $6 \mathrm{ml}$ of air into the bag at a flow rate of $2 \mathrm{ml} / \mathrm{min}$. The intrabag pressure was recorded using a pressure amplifier (BP Amp; AD Instruments Pty. Ltd.) and a data acquisition device (PowerLab 4/26; AD Instruments Pty. Ltd.). The baseline intrabag pressure for 1 minute was measured 5 minutes after the start of air injection. The air was then withdrawn from the bag and the animals were allowed to rest for about 10 minutes. After that, a liquid meal ( $4 \mathrm{ml}, 1.7 \mathrm{kcal})$ was orally administered using a gavage needle (RZ-2; CLEA Japan, Inc., Tokyo, Japan). The liquid meal consisted of powdered standard laboratory chow [CG-7; CLEA Japan, Inc. (278.1 kcal, 18.1\%, w/w, crude protein; $3.4 \%$, w/w, crude fat; and $16.8 \%$, w/w, crude fiber per $100 \mathrm{~g})]$ suspended in distilled water (DW) at $15 \%(\mathrm{w} / \mathrm{v})$ using a Polytron homogenizer. Immediately after administration of the liquid meal, $6 \mathrm{ml}$ air was again injected into the bag, and the intrabag pressure for 1 minute was recorded every 5 minutes until 30 minutes after the start of air injection. The mean change in intrabag pressure (mean $\Delta$ intrabag pressure), which is the average of changes in intrabag pressure from the basal level at 5-minute intervals, was calculated using LabChart 6 (AD Instruments Pty. Ltd.). In this study, each guinea pig was used up to three times. When used for multiple measurements, the animals were rested for a minimum of 3 days between measurements. The baseline intrabag pressure was determined before every measurement and if it was low $(<4.0 \mathrm{~mm} \mathrm{Hg} / \mathrm{min})$, the animal was not used. The animals were sacrificed at the end of the study and the integrity and location of the bags were evaluated. Extremely low intrabag pressures were detected when the bag was damaged or dislocated to the distal stomach. The animals with dislocated or damaged bags were excluded from the study.

Administration of Test Samples. DB $(0.02 \mathrm{nmol} / \mathrm{ml}$ to $60 \mu \mathrm{mol} / \mathrm{ml}$ ) was dissolved in DW. RKT (100 or $200 \mathrm{mg} / \mathrm{ml}$ : no toxicologically meaningful change was observed in singledose toxicity studies in rats) and its ingredients liquiritigenin $(0.2-0.4 \mathrm{mg} / \mathrm{ml})$ and naringenin $(0.4-0.8 \mathrm{mg} / \mathrm{ml})$ were suspended in DW. DB $(0.02 \mathrm{nmol} / \mathrm{ml}-2 \mu \mathrm{mol} / \mathrm{ml})$, RKT, liquiritigenin, or naringenin was administered into the oral cavity $(0.5 \mathrm{ml} / \mathrm{kg})$. Fifteen minutes later, the liquid meal was orally administered as described above, to induce physiologic GA. In addition, DB $(0.2 \mathrm{nmol} / \mathrm{ml}$ to $60 \mu \mathrm{mol} / \mathrm{ml})$ or RKT $(200 \mathrm{mg} / \mathrm{ml})$ was administered via the stomach $(0.5$ or $5 \mathrm{ml} / \mathrm{kg}$, respectively) and the liquid meal was orally administered 30 minutes later.

Taste Measurement. DB $(0.1-100 \mathrm{nmol} / \mathrm{ml})$ was dissolved in DW; $\mathrm{KCl}$ was added to the solution to a final concentration of $10 \mathrm{mM}$. RKT $(200 \mathrm{mg} / \mathrm{ml})$ and its ingredients liquiritigenin $(0.4 \mathrm{mg} / \mathrm{ml})$ and naringenin $(0.8 \mathrm{mg} / \mathrm{ml})$ were suspended in $\mathrm{DW}$; $\mathrm{KCl}$ was added to the solution to a final concentration of $10 \mathrm{mM}$. After centrifugation, the supernatant was collected and used as the test samples. The test samples were analyzed for their prominent taste using the tastesensing system SA402B (Intelligent Sensor Technology, Inc.). This system is equipped with seven artificial lipid membrane sensor probes that can detect the taste factors of anionic bitterness, aftertaste of anionic bitterness, aftertaste of catatonic bitterness 1 , aftertaste of catatonic bitterness 2 , astringency, aftertaste of astringency, umami, aftertaste of umami, saltiness, and acidity (Table 1). Taste intensity was estimated from the outputs of the artificial lipid membrane sensor probes, the basis of which is Weber-Fechner's law that the intensity of perception is proportional to the logarithm of stimulus intensity (Uchida et al., 2003; Kobayashi et al., 2010; Tahara et al., 2011).

Statistical Analysis. All data are expressed as mean \pm S.E. To assess the differences in intrabag pressure among groups, a Student's $t$ test, Dunnett's test was performed using StatLight (Yukms Co., Ltd., Tokyo, Japan). Values of $P<0.05$ were considered statistically significant.

\section{Results}

Effects of DB Administration on GA. The time course of intrabag pressure after liquid meal administration in guinea pigs is shown in Fig. 1A. The intrabag pressure in the distilled water-treated group was temporally decreased 5 or 10 minutes after liquid meal administration and then gradually increased. In guinea pigs, intraoral administration of DB

TABLE 1

Characteristics of taste information on taste sensors

\begin{tabular}{ll}
\hline Sensor Probes & \multicolumn{1}{c}{ Taste Factor } \\
\hline C00 & Anionic bitterness \\
& Aftertaste of anionic bitterness \\
AC0 & Aftertaste of cationic bitterness $1^{a}$ \\
AN0 & Aftertaste of cationic bitterness $2{\text { (mineral })^{a}}$ \\
AE1 & Astringency \\
& Aftertaste of astringency \\
AAE & Umami \\
& Aftertaste of umami \\
CT0 & Saltiness \\
CA0 & Acidity \\
\hline
\end{tabular}

${ }^{a}$ Converted from change of membrane potential caused by absorption (CPA) values of each sensor probe; others converted from relative potentials. 




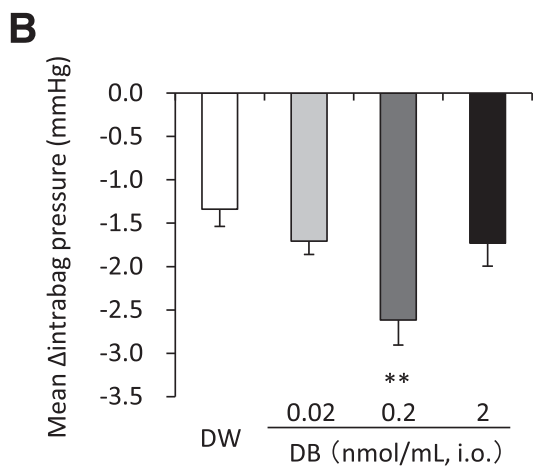

Fig. 1. Influence of intraoral administration of denatonium benzoate $(\mathrm{DB})$ on gastric accommodation in guinea pigs. (A) Time course of intrabag pressure after liquid meal administration in guinea pigs. (B) The mean $\Delta$ intrabag pressure was decreased by $\mathrm{DB}(0.2 \mathrm{nmol} / \mathrm{ml})$ administration, compared with that in the distilled water-treated group. Data are expressed as mean \pm S.E. $(n=5-7)$. ** $P<0.01$, *** $P<0.001$ (A: vs. pre, B: vs. DW, by Dunnett's test). i.o.; intraoral administration. decreased the intrabag pressure 5 minutes after liquid meal administration in all groups (Fig. 1A). As shown in Fig. 1B, the decrease in the mean change in intrabag pressure was significantly promoted by $0.2 \mathrm{nmol} / \mathrm{ml}$ i.o. DB treatment, but not by other doses of DB (0.02 or $2 \mathrm{nmol} / \mathrm{ml}$, i.o.), compared with the DW-treatment group (DW: $-1.34 \pm 0.20 \mathrm{~mm} \mathrm{Hg}$, DB $0.2 \mathrm{nmol} / \mathrm{ml}:-2.62 \pm 0.29 ; P<0.01$ by Dunnett's test).

A decrease in intrabag pressure was also observed in the guinea pigs that received intragastric DB (Fig. 2A). As shown in Fig. 2B, the mean change in intrabag pressure, which is calculated as the change in intrabag pressure from the basal level, significantly decreased in the intragastric DB (0.1 and $1 \mathrm{nmol} / \mathrm{kg}$ )-treated group, compared with the DW-treated group (DW: $-1.85 \pm 0.15 \mathrm{~mm} \mathrm{Hg}$, DB $0.1 \mathrm{nmol} / \mathrm{kg}:-2.66 \pm$ $0.18 \mathrm{~mm} \mathrm{Hg}, P<0.05$, DB $1 \mathrm{nmol} / \mathrm{kg}:-2.85 \pm 0.23 \mathrm{~mm} \mathrm{Hg}$, $P<0.01$ by Dunnett's test). These results indicate that GA was enhanced by intragastric administration of the potent TAS2Rs agonist DB in guinea pigs. However, the effect was attenuated at a higher DB dose $(10 \mathrm{nmol} / \mathrm{kg}$, i.g. $)$. The treatment with DB at higher doses (30 $\mu \mathrm{mol} / \mathrm{kg}$, i.g.) significantly suppressed the decrease in the mean change in intrabag pressure (DW: $-1.80 \pm 0.18 \mathrm{~mm} \mathrm{Hg}, \mathrm{DB}:-1.17 \pm 0.21$, $P<0.05$ by Student's $t$ test) (Fig. 2C).

Effects of RKT and Its Flavonoids on GA. Intraoral administration of RKT $(200 \mathrm{mg} / \mathrm{ml})$ in guinea pigs significantly enhanced the mean change in intrabag pressure, compared with DW administration (DW: $-1.26 \pm 0.13 \mathrm{~mm}$ Hg, RKT: $-2.46 \pm 0.32, P<0.01$ by Dunnett's test) (Fig. 3A). Additionally, a promoting effect was also observed by intragastric administration of RKT at a dose of $1000 \mathrm{mg} / \mathrm{kg}$ (DW: $-1.08 \pm 0.16 \mathrm{~mm} \mathrm{Hg}, \mathrm{RKT}:-2.01 \pm 0.29, P<0.05$ by Student's $t$ test) (Fig. 3B).

The effects of liquiritigenin and naringenin, which are components of RKT and have TAS2R-agonistic activity, on the mean change in intrabag pressure after liquid meal administration were assessed. Intraoral administration of $0.4 \mathrm{mg} / \mathrm{ml}$ liquiritigenin significantly promoted the decrease in the mean change in intrabag pressure (DW: $-1.23 \pm 0.12 \mathrm{~mm} \mathrm{Hg}$,
A
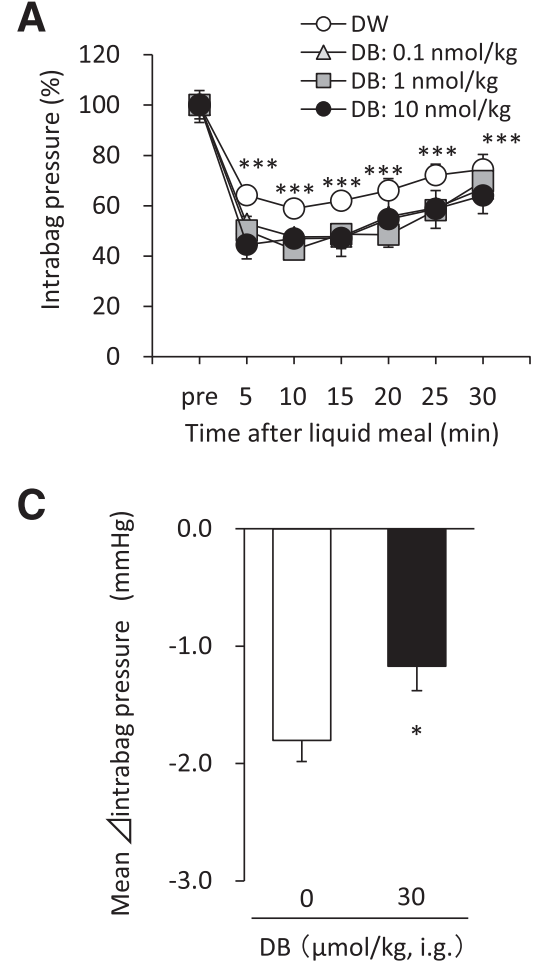

B

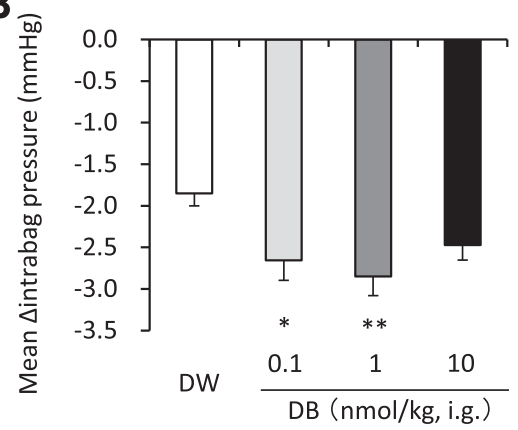

Fig. 2. Influence of intragastric administration of denatonium benzoate (DB) on gastric accommodation in guinea pigs. (A) Time course of intrabag pressure after liquid meal administration in guinea pigs. (B) The mean $\Delta$ intrabag pressure (the changes in intrabag pressure from basal level) was decreased by $\mathrm{DB}(0.1$ and $1 \mathrm{nmol} / \mathrm{kg}$ ) administration, compared with that in the distilled water-treated group. (C) The treatment with DB $(30 \mu \mathrm{mol} / \mathrm{kg})$ suppressed the decrease in the mean $\Delta$ intrabag pressure. Data are expressed as mean \pm S.E. $(n=5)$. $P<0.05$, ** $P<0.01$, *** $P<0.001$ (A: vs. pre, B: vs. DW, by Dunnett's test, C: Student's t-test). i.g.; intragastric administration. 


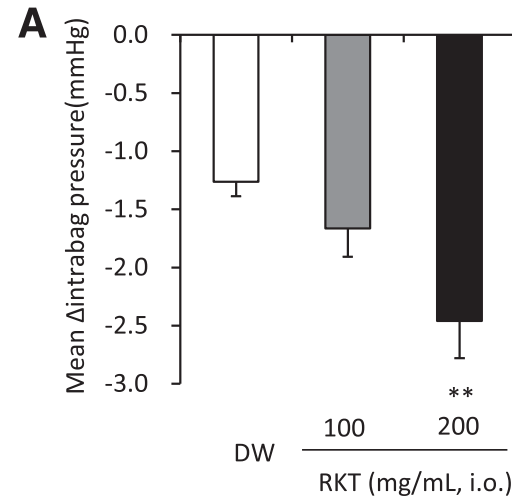

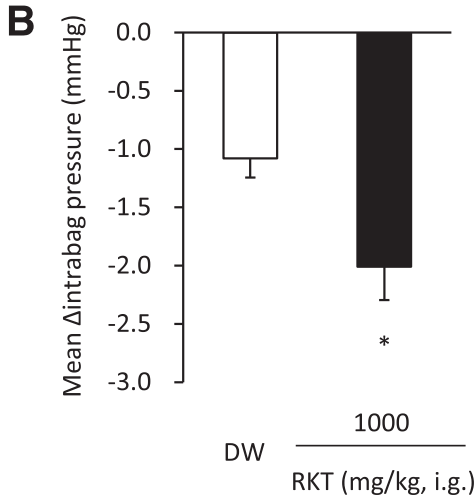

Fig. 3. Influence of rikkunshito (RKT) on gastric accommodation in guinea pigs. Intraoral (A) and intragastric (B) administration of RKT in guinea pigs enhanced the decrease in the mean $\Delta$ intrabag pressure. Data are expressed as mean \pm S.E. $(n=6) . * P<0.05 ; * * P<0.01$ (A: Dunnett's test, B: Student's $t$ test). DW, distilled water; i.o., intraoral administration; i.g., intragastric administration. liquiritigenin: $-1.89 \pm 0.13 ; P<0.01$ by Dunnett's test) (Fig. 4A). Intraoral administration of $0.8 \mathrm{mg} / \mathrm{ml}$ naringenin significantly promoted the decrease in the mean change in intrabag pressure (DW: $-1.29 \pm 0.22 \mathrm{~mm} \mathrm{Hg}$, naringenin: $-2.34 \pm 0.28, P<0.05$ by Dunnett's test) (Fig. $4 \mathrm{~B}$ ).

Taste Patterns of Test Samples. Ten taste factors of the test samples were measured using a taste-sensing system. As shown in Fig. 5, the aftertastes of cationic bitterness 1 and cationic bitterness 2 were detected in DB solution (10 and $100 \mathrm{nmol} / \mathrm{ml}$ ). The taste intensity increased in a dose-dependent manner. Other taste factors were not detectable. As shown in Table 2, the taste intensity of anionic bitterness was the highest of the 10 taste factors in liquiritigenin $(0.4 \mathrm{mg} / \mathrm{ml})$ and naringenin $(0.8 \mathrm{mg} / \mathrm{ml})$ solutions. In contrast, almost all taste factors were detected in the RKT (200 $\mathrm{mg} / \mathrm{ml})$ solution. Among them, a remarkable increase in the aftertaste of cationic bitterness 2 was observed.

\section{Discussion}

We found that the intraoral or intragastric administration of the bitter compound DB promoted GA in guinea pigs. DB has been found to bind to eight of the TAS2R subtypes and has TAS2Rs agonist activities in human (Meyerhof et al., 2010). TAS2Rs are expressed in taste receptor cells assembled into taste buds on the tongue, enteroendocrine cells, and smooth muscle cells of the GI tract. Several studies have demonstrated that TAS2Rs are coexpressed with the signal element taste-specific $\mathrm{G}$ protein gustducin, and involved in regulating gut hormone release and GI motility in humans and animals (Wu et al., 2002; Shi et al., 2003; Chen et al., 2006; Rozengurt and Sternini, 2007; Hao et al., 2008; Jeon et al., 2008; Janssen et al., 2011; Daly et al., 2013). Therefore, the GA-promoting effect of DB could be mediated by a gut hormone or a direct action on smooth muscle cells in guinea pigs.

The present study showed that intraoral administration of DB induced GA-promotion in guinea pigs. Taste stimulation of taste buds on the tongue simultaneously activates GI motility and stimulates the secretion of gastric acids, gastrin, and pancreatic polypeptide 2 . Recent evidence suggests that bitter-tasting plants elicit cephalic responses through the oropharyngeal TAS2Rs and facilitate digestive activity (McMullen et al., 2015). Moreover, it has been demonstrated that $\mathrm{G}$ protein gustducin, which is an important first signal transmitter of taste signals to the brain (Behrens and Meyerhof, 2011), is extensively coexpressed with TAS2Rs in the soft palate and fungiform papillae on the tongue
(Tomonari et al., 2012), and that motilin secreted from the thyroid into the peripheral plasma by paraventricular nucleus stimulation affects gastric motility (Guo et al., 2011). Additionally, motilin also is a gut hormone released from the endocrine cells of the duodenal and jejunal mucosa as a regulator of gastrointestinal motility (Guo et al., 2011). Motilin infusion caused a contraction of the proximal stomach in humans, resulting in the inhibition of GA induced by satiety drinking (Cuomo et al., 2006). It has also been reported that taste stimulation accelerates the efferent activity of the gastric branch of the vagus nerve (Niijima, 1991; Niot and Besnard, 2017). Therefore, the promoting
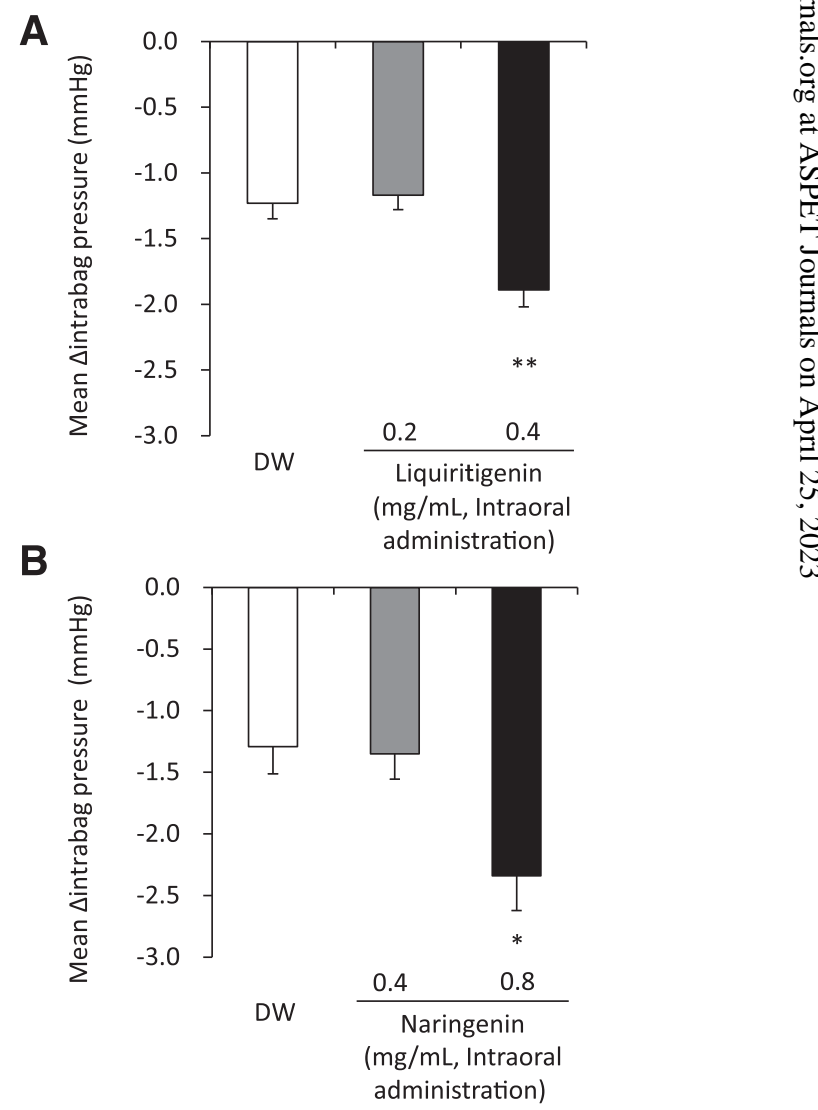

Fig. 4. Influence of the ingredients of rikkunshito on gastric accommodation in guinea pigs. Intraoral administration of liquiritigenin (A) and naringenin (B) in guinea pigs enhanced the decrease in the mean $\Delta$ intrabag pressure. Data are expressed as mean \pm S.E. $(n=6$ to 7$)$. $* P<0.05 ; * * P<0.01$ (Dunnett's test). 


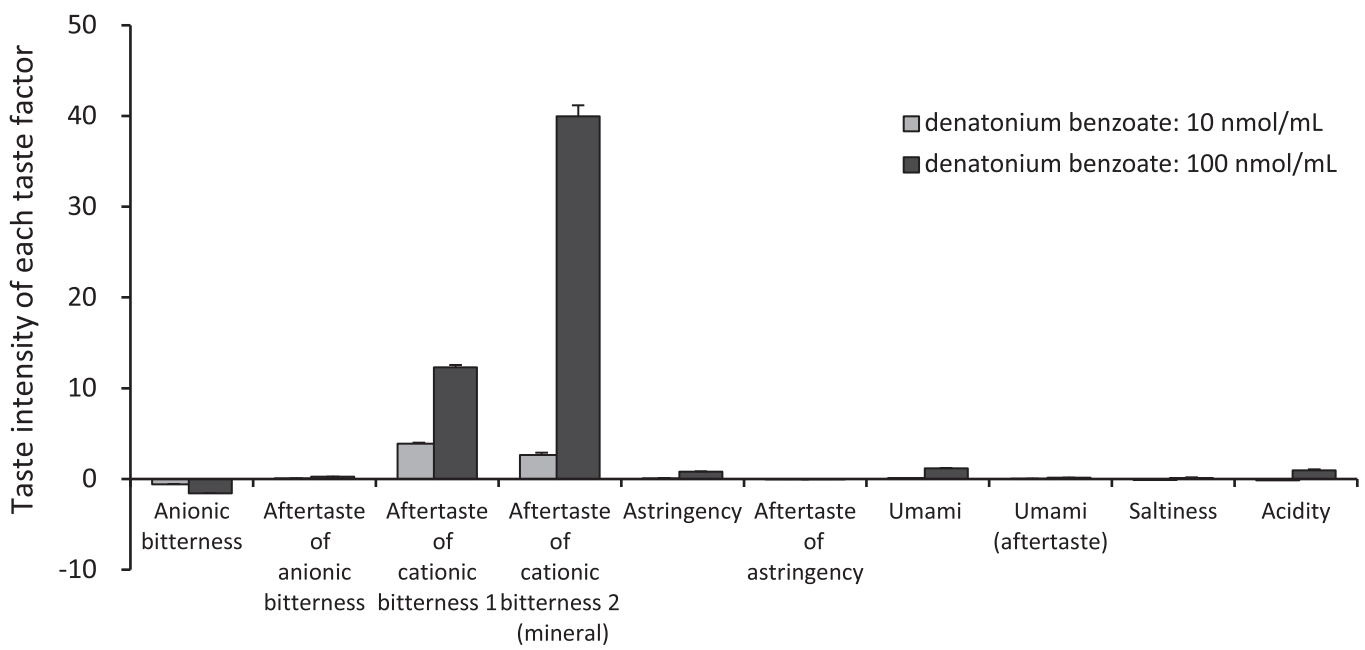

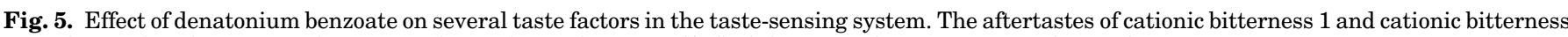
were detected in denatonium benzoate solution (10 and $100 \mathrm{nmol} / \mathrm{ml})$. Other taste factors were not detected.

effect of DB on GA in guinea pigs could probably be involved in regulating motilin secretion or a direct action on smooth muscles through the cephalic phase responses.

In healthy female volunteers, intragastric administration of DB inhibited the increase in plasma motilin levels after an overnight fast and reduced the fluctuation of antral motility (Deloose et al., 2017; Deloose et al., 2018). Therefore, it was suggested that the promoting effect of DB on GA was also mediated by the decreased release of motilin through TAS2Rs in the gut. In addition, cellular studies indicated that the activation of TAS2Rs with DB induced the secretion of glucagon-like peptide-1 (GLP-1) from enteroendocrine cells (Kim et al., 2014). The increase in GLP-1 secretion by DB was also observed in db/db mice (van Avesaat et al., 2015). GLP-1 causes a reduction in cholinergic contractions in the GI smooth muscles by acting on the enteric nervous system in the mouse intestine (Amato et al., 2010; Rotondo et al., 2011). These findings suggest that the enhancement of GA by intragastric administration of DB in guinea pigs might be mediated by the regulation of GLP-1 release from the enteroendocrine cells.

On the contrary, intraoral and intragastric treatment with DB at a higher dose did not promote GA. Furthermore, intragastric treatment with DB at a higher dose $(30 \mu \mathrm{mol} / \mathrm{kg})$ significantly suppressed GA in guinea pigs in this study. It was reported that thresholds for the bitter taste in foods can be greater than, equal to, or less than those for toxicity and that the bitter rejection response is maladaptive for omnivores or herbivores because it would cause them to limit drastically the range of potential foods (Glendinning, 1994). Therefore, the bitter substance can be rejected at high concentrations above the threshold. It was also demonstrated that DB induced contraction in mouse gastric fundic smooth muscle strips by an increase in intracellular calcium concentration in smooth muscle cells (Avau et al., 2015). Furthermore, intragastric DB (1 $\mu \mathrm{mol} / \mathrm{kg}$ ) administration impaired the relaxation of the fundus in response to nutrient infusion and increased the satiation in healthy volunteers (Avau et al., 2015). These reports are similar to the effect of intragastric treatment with DB at a higher dose $(30 \mu \mathrm{mol} / \mathrm{kg})$ on GA in guinea pigs, although the sensitivity to bitter stimulants seems to be different between humans and guinea pigs. TAS2R subtypes TAS2R5, 10, and 14 reportedly have a predominant role in inducing relaxation of smooth muscle cells (Grassin-Delyle et al., 2013). DB has binding activities against TAS2R10 (Meyerhof et al., 2010). These findings suggest that the optimal dose of DB for binding TAS2Rs might differ between TAS2R subtypes. Hence, low-dose DB-induced stimulation of TAS2Rs may have enhanced GA in guinea pigs, which is different from the effect of high-dose DB administration. Therefore, it may be considered that DB regulated GA by several mechanisms associated with TAS2Rs. In addition, it may be important to evaluate gastric emptying following GA.

TABLE 2

Taste intensities of each taste factor in each sample

\begin{tabular}{|c|c|c|c|}
\hline \multirow{2}{*}{ Taste Factors } & \multicolumn{3}{|c|}{ Taste Intensities of Each Taste Factor (Mean \pm S.E.M.) } \\
\hline & RKT (200 mg/ml) & Liquiritigenin $(0.4 \mathrm{mg} / \mathrm{ml})$ & Naringenin $(0.8 \mathrm{mg} / \mathrm{ml})$ \\
\hline Anionic bitterness & $5.77 \pm 0.05$ & $1.57 \pm 0.07$ & $1.41 \pm 0.15$ \\
\hline Aftertaste of anionic bitterness & $5.30 \pm 0.06$ & $0.33 \pm 0.00$ & $0.82 \pm 0.03$ \\
\hline Aftertaste of cationic bitterness 1 & $7.32 \pm 0.18$ & $-0.16 \pm 0.06$ & $-0.27 \pm 0.13$ \\
\hline Aftertaste of cationic bitterness 2 (mineral) & $64.41 \pm 0.57$ & $-0.41 \pm 0.12$ & $-0.61 \pm 0.41$ \\
\hline Astringency & $5.96 \pm 0.15$ & $0.44 \pm 0.01$ & $0.25 \pm 0.01$ \\
\hline Aftertaste of astringency & $3.28 \pm 0.07$ & $0.33 \pm 0.01$ & $0.22 \pm 0.01$ \\
\hline Umami & $2.71 \pm 0.15$ & $0.12 \pm 0.01$ & $0.13 \pm 0.04$ \\
\hline Aftertaste of umami & $5.19 \pm 0.16$ & $0.14 \pm 0.01$ & $0.16 \pm 0.01$ \\
\hline Saltiness & $10.78 \pm 0.24$ & $0.07 \pm 0.01$ & $-0.01 \pm 0.01$ \\
\hline Acidity & $0.20 \pm 0.24$ & $-0.27 \pm 0.01$ & $-0.48 \pm 0.09$ \\
\hline
\end{tabular}


Avau et al. (2015) have demonstrated that intragastric administration of DB ( $60 \mu \mathrm{mol} / \mathrm{kg}$, by mouth) inhibited gastric emptying rate $(\mathrm{GE})$ in mice. TAS2R stimulation is also thought to affect GE. However, it is unclear whether the regulation of GA by DB is involved in the suppression of GE.

Several studies have demonstrated that RKT enhances GA in humans and animals. In a study of GA reflex in FD patients using extracorporeal ultrasonography, the expansion rate of the proximal stomach after ingestion of a liquid meal was increased by RKT treatment (Kusunoki et al., 2010). The proximal gastric volume increased immediately after liquid infusion in conscious dogs, and the effect of RKT was significantly greater than that of water (Furukawa et al., 2013). In this study, intraoral and intragastric RKT-treated guinea pigs exhibited a potentiated physiologic GA. Furthermore, the enhancement of GA in guinea pigs was observed after intraoral administration of the components of RKT, liquiritigenin and naringenin. These components reportedly have agonistic effects on TAS2R14 and TAS2R39 (Rouzade et al., 1998), suggesting that RKT could regulate GA by stimulating TAS2R signaling in guinea pigs.

Recent evidence has suggested that regulation of gastrointestinal function may also be affected by the taste sensory system (Loper et al., 2015). Taste stimulants, except for the bitter taste, also regulate gut hormone release and GI motility (Lavin et al., 2002; Kendig et al., 2014). The assessment of taste function using a taste-sensing system demonstrated that liquiritigenin and naringenin showed the highest-intensity bitterness among all tastes. However, the type of bitterness of these components was different from that of DB, suggesting that different TAS2R subtypes were involved. These results indicated that liquiritigenin and naringenin specifically stimulated the TAS2Rs and that other taste receptors may not be involved in these effects on GA in guinea pigs. Our results suggest that TAS2R may be involved in the regulation of gastrointestinal motility. We speculate that the modulation of TAS2R activity could be a novel therapeutic approach for functional gastrointestinal disorders such as FD, gastroesophageal reflux disease, and nonerosive reflux disease.

In conclusion, bitter stimulants regulated GA in guinea pigs, which may have been mediated by TAS2R activation in the oral cavity and gut. However, the mechanisms of GA activation mediated through the cephalic phase responses or gut hormone are not well understood and need further investigation.

\section{Acknowledgments}

We appreciate the guidance given by Dr. H. Miwa on the gastric accommodation evaluation system using guinea pigs and for his helpful technical advice.

\section{Authorship Contributions}

Participated in research design: Harada, Fujitsuka.

Conducted experiments: Harada, Koseki, Sekine.

Performed data analysis: Harada, Fujitsuka.

Wrote or contributed to the writing of the manuscript: Harada, Fujitsuka, Kobayashi.

\section{References}

Amato A, Cinci L, Rotondo A, Serio R, Faussone-Pellegrini MS, Vannucchi MG, and Mulè F (2010) Peripheral motor action of glucagon-like peptide-1 through enteric neuronal receptors. Neurogastroenterol Motil 22:664-e203.

Avau B, Rotondo A, Thijs T, Andrews CN, Janssen P, Tack J, and Depoortere I (2015) Targeting extra-oral bitter taste receptors modulates gastrointestinal motility with effects on satiation. Sci Rep 5:15985.
Barretto RP, Gillis-Smith S, Chandrashekar J, Yarmolinsky DA, Schnitzer MJ, Ryba NJ, and Zuker CS (2015) The neural representation of taste quality at the periphery. Nature 517:373-376.

Behrens M and Meyerhof W (2011) Gustatory and extragustatory functions of mammalian taste receptors. Physiol Behav 105:4-13.

Cellini J, DiNovo K, Harlow J, and LePard KJ (2011) Regional differences in neostigmine-induced contraction and relaxation of stomach from diabetic guinea pig. Auton Neurosci 160:69-81.

Chandrashekar J, Mueller KL, Hoon MA, Adler E, Feng L, Guo W, Zuker CS, and Ryba NJ (2000) T2Rs function as bitter taste receptors. Cell 100:703-711.

Chen MC, Wu SV, Reeve JR Jr, and Rozengurt E (2006) Bitter stimuli induce Ca2+ signaling and CCK release in enteroendocrine STC-1 cells: role of L-type voltagesensitive Ca2+ channels. Am J Physiol Cell Physiol 291:C726-C739.

Cuomo R, Vandaele P, Coulie B, Peeters T, Depoortere I, Janssens J, and Tack J (2006) Influence of motilin on gastric fundus tone and on meal-induced satiety in man: role of cholinergic pathways. Am J Gastroenterol 101:804-811.

Daly K, Al-Rammahi M, Arora DK, Moran AW, Proudman CJ, Ninomiya Y, and Shirazi-Beechey SP (2012) Expression of sweet receptor components in equine small intestine: relevance to intestinal glucose transport. Am J Physiol Regul Integr Comp Physiol 303:R199-R208.

Daly K, Al-Rammahi M, Moran A, Marcello M, Ninomiya Y, and Shirazi-Beechey SP (2013) Sensing of amino acids by the gut-expressed taste receptor T1R1-T1R3 stimulates CCK secretion. Am $J$ Physiol Gastrointest Liver Physiol 304: G271-G282.

Deloose E, Corsetti M, Van Oudenhove L, Depoortere I, and Tack J (2018) Intragastric infusion of the bitter tastant quinine suppresses hormone release and antral motility during the fasting state in healthy female volunteers. Neurogastroenterol Motil 30:e13171.

Deloose E, Janssen P, Corsetti M, Biesiekierski J, Masuy I, Rotondo A, Van Oudenhove L, Depoortere I, and Tack J (2017) Intragastric infusion of denatonium benzoate attenuates interdigestive gastric motility and hunger scores in healthy female volunteers. Am J Clin Nutr 105:580-588.

Depoortere I (2014) Taste receptors of the gut: emerging roles in health and disease. Gut 63:179-190.

Furukawa N, Manabe N, Kase Y, Hattori T, Imamura H, Kusunoki H, and Haruma K (2013) Intragastric infusion of rikkunshito (kampo) induces proximal stomach relaxation in conscious dogs. Auton Neurosci 179:14-22.

Glendinning JI (1994) Is the bitter rejection response always adaptive? Physiol Behav 56:1217-1227.

Grassin-Delyle S, Abrial C, Fayad-Kobeissi S, Brollo M, Faisy C, Alvarez JC, Naline E, and Devillier P (2013) The expression and relaxant effect of bitter taste receptors in human bronchi. Respir Res 14:134.

Guo F, Xu L, Sun X, Gao S, and Zhu H (2011) The paraventricular nucleus modulates thyroidal motilin release and rat gastric motility. $J$ Neuroendocrinol 23:767-777.

Hao S, Sternini C, and Raybould HE (2008) Role of CCK1 and Y2 receptors in activation of hindbrain neurons induced by intragastric administration of bitter taste receptor ligands. Am J Physiol Regul Integr Comp Physiol 294:R33-R38.

Ikeo K, Oshima T, Sei H, Kondo T, Fukui H, Watari J, and Miwa H (2017) Acotiamide improves stress-induced impaired gastric accommodation. Neurogastroenterol Motil 29:e12991.

Janssen S and Depoortere I (2013) Nutrient sensing in the gut: new roads to therapeutics? Trends Endocrinol Metab 24:92-100.

Janssen S, Laermans J, Verhulst PJ, Thijs T, Tack J, and Depoortere I (2011) Bitter taste receptors and $\alpha$-gustducin regulate the secretion of ghrelin with functional effects on food intake and gastric emptying. Proc Natl Acad Sci USA 108: 2094-2099.

Jeon TI, Zhu B, Larson JL, and Osborne TF (2008) SREBP-2 regulates gut peptide secretion through intestinal bitter taste receptor signaling in mice. $J$ Clin Invest 118:3693-3700.

Ji SW, Park HJ, Cho JS, Lim JH, and Lee SI (2003) Investigation into the effects of mosapride on motility of Guinea pig stomach, ileum, and colon. Yonsei Med J 44: 653-664.

Katschinski M (2000) Nutritional implications of cephalic phase gastrointestinal responses. Appetite 34:189-196

Kendig DM, Hurst NR, Bradley ZL, Mahavadi S, Kuemmerle JF, Lyall V, DeSimone J, Murthy KS, and Grider JR (2014) Activation of the umami taste receptor (T1R1/T1R3) initiates the peristaltic reflex and pellet propulsion in the distal colon. Am J Physiol Gastrointest Liver Physiol 307:G1100-G1107.

Kim KS, Egan JM, and Jang HJ (2014) Denatonium induces secretion of glucagonlike peptide-1 through activation of bitter taste receptor pathways. Diabetologia 57:2117-2125.

Kindt S and Tack J (2006) Impaired gastric accommodation and its role in dyspepsia. Gut 55:1685-1691.

Kobayashi Y, Habara M, Ikezazki H, Chen R, Naito Y, and Toko K (2010) Advanced taste sensors based on artificial lipids with global selectivity to basic taste qualities and high correlation to sensory scores. Sensors (Basel) 10:3411-3443.

Koseki J, Oshima T, Kondo T, Tomita T, Fukui H, Watari J, Hattori T, Kase Y, and Miwa H (2012) Role of transient receptor potential ankyrin 1 in gastric accommodation in conscious guinea pigs. J Pharmacol Exp Ther 341:205-212.

Kusunoki H, Haruma K, Hata J, Ishii M, Kamada T, Yamashita N, Honda K, Inoue $\mathrm{K}$, Imamura H, Manabe N, et al. (2010) Efficacy of Rikkunshito, a traditional Japanese medicine (Kampo), in treating functional dyspepsia. Intern Med 49: 2195-2202

Lavin JH, French SJ, and Read NW (2002) Comparison of oral and gastric administration of sucrose and maltose on gastric emptying rate and appetite. Int $J$ Obes Relat Metab Disord 26:80-86.

Loper HB, La Sala M, Dotson C, and Steinle N (2015) Taste perception, associated hormonal modulation, and nutrient intake. Nutr Rev 73:83-91.

McMullen MK, Whitehouse JM, and Towell A (2015) Bitters: time for a new paradigm. Evid Based Complement Alternat Med 2015:670504. 
Meyerhof W, Batram C, Kuhn C, Brockhoff A, Chudoba E, Bufe B, Appendino G, and Behrens M (2010) The molecular receptive ranges of human TAS2R bitter taste receptors. Chem Senses 35:157-170.

Miwa H, Koseki J, Oshima T, Hattori T, Kase Y, Kondo T, Fukui H, Tomita T, Ohda $\mathrm{Y}$, and Watari $\mathrm{J}$ (2016) Impairment of gastric accommodation induced by wateravoidance stress is mediated by 5-HT2B receptors. Neurogastroenterol Motil 28: $765-778$.

Niijima A (1991) Effects of oral and intestinal stimulation with umami substance on gastric vagus activity. Physiol Behav 49:1025-1028.

Niot I and Besnard P (2017) Appetite control by the tongue-gut axis and evaluation of the role of CD36/SR-B2. Biochimie 136:27-32.

Roland WS, van Buren L, Gruppen H, Driesse M, Gouka RJ, Smit G, and Vincken JP (2013) Bitter taste receptor activation by flavonoids and isoflavonoids: modeled structural requirements for activation of hTAS2R14 and hTAS2R39. J Agric Food Chem 61:10454-10466.

Rotondo A, Amato A, Lentini L, Baldassano S, and Mulè F (2011) Glucagon-like peptide-1 relaxes gastric antrum through nitric oxide in mice. Peptides 32:60-64.

Rouzade ML, Fioramonti J, and Bueno L (1998) Decrease in gastric sensitivity to distension by 5-HT1A receptor agonists in rats. Dig Dis Sci 43:2048-2054.

Rozengurt E and Sternini C (2007) Taste receptor signaling in the mammalian gut. Curr Opin Pharmacol 7:557-562.

Shi P, Zhang J, Yang H, and Zhang YP (2003) Adaptive diversification of bitter taste receptor genes in Mammalian evolution. Mol Biol Evol 20:805-814.

Shiratori M, Shoji T, Kanazawa M, Hongo M, and Fukudo S (2011) Effect of rikkunshito on gastric sensorimotor function under distention. Neurogastroenterol Motil 23:323-329, e155-e156

Tack J, Demedts I, Meulemans A, Schuurkes J, and Janssens J (2002) Role of nitric oxide in the gastric accommodation reflex and in meal induced satiety in humans. Gut 51:219-224.

Tack J, Piessevaux H, Coulie B, Caenepeel P, and Janssens J (1998) Role of impaired gastric accommodation to a meal in functional dyspepsia. Gastroenterology $\mathbf{1 1 5}$ 1346-1352.

Tahara Y, Ikeda A, Maehara Y, Habara M, and Toko K (2011) Development and evaluation of a miniaturized taste sensor chip. Sensors (Basel) 11:9878-9886.
Tominaga K, Sakata Y, Kusunoki H, Odaka T, Sakurai K, Kawamura O, Nagahara A, Takeuchi T, Fujikawa Y, Oshima T, et al. (2018) Rikkunshito simultaneously improves dyspepsia correlated with anxiety in patients with functional dyspepsia: a randomized clinical trial (the DREAM study). Neurogastroenterol Motil $\mathbf{3 0}$ e13319.

Tomonari H, Miura H, Nakayama A, Matsumura E, Ooki M, Ninomiya Y, and Harada S (2012) G $\alpha$-gustducin is extensively coexpressed with sweet and bitter taste receptors in both the soft palate and fungiform papillae but has a different functional significance. Chem Senses 37:241-251.

Tsai CC, Tey SL, Lee MC, Liu CW, Su YT, and Huang SC (2018) Mechanism of resveratrol-induced relaxation of the guinea pig fundus. Phytomedicine 43:55-59.

Uchida T, Tanigake A, Miyanaga Y, Matsuyama K, Kunitomo M, Kobayashi Y, Ikezaki H, and Taniguchi A (2003) Evaluation of the bitterness of antibiotics using a taste sensor. J Pharm Pharmacol 55:1479-1485.

van Avesaat M, Troost FJ, Ripken D, Peters J, Hendriks HF, and Masclee AA (2015) Intraduodenal infusion of a combination of tastants decreases food intake in humans. Am J Clin Nutr 102:729-735.

van den Elzen BD and Boeckxstaens GE (2006) Review article: a critical view on impaired accommodation as therapeutic target for functional dyspepsia. Aliment Pharmacol Ther 23:1499-1510.

Wu SV, Rozengurt N, Yang M, Young SH, Sinnett-Smith J, and Rozengurt E (2002) Expression of bitter taste receptors of the T2R family in the gastrointestinal tract and enteroendocrine STC-1 cells. Proc Natl Acad Sci USA 99:2392-2397.

Youn YH, Choi EJ, Lee YH, Oshima T, Miwa H, and Park H (2015) The effects of 5 -hydroxytryptamine1a receptor agonist, buspirone on the gastric fundus accommodation in an animal model using guinea pigs. Neurogastroenterol Motil 27: $532-541$.

Address correspondence to: Dr. Yumi Harada, Tsumura Kampo Research Laboratories, Tsumura \& Co., 3586 Yoshiwara, Ami-machi, Inashiki-Gun, Ibaraki 300-1192, Japan. E-mail: harada_yumi@mail.tsumura.co.jp; or Hitomi Sekine, sekine_hitomi@mail.tsumura.co.jp; or Naoki Fujitsuka, fujitsuka_naoki@ mail.tsumura.co.jp; or Hiroyuki Kobayashi, koba@juntendo.ac.jp 